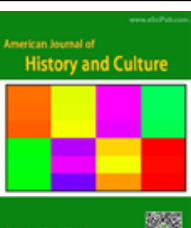

\title{
American Journal of History and Culture (DOI:10.28933/AJHC)
}

\section{Dream of Having Superpowers: Comparison between Good and Bad Superpowers in Real Life as a Person According to Science}

\section{Farhana Haque}

Brac University, Mohakhali, Dhaka,Bangladesh

\section{ABSTRACT}

To acquire any kinds of superpowers, most of the people are absolutely desperate. Inside of their mind they actually use to dream about helping the entire human race by using their superpowers. Here i have the intention to elucidate this matter of having or dreaming about superpowers with different logics. As we know that, according to science human have a fabulous body mechanism with genius super computer brain, which can accumulate, scan and then send essential conscious and unconscious orders/directions to other parts of human body. On this point, i would like to add about Rick Hanson's statements. He denotes, " On average each of the 1000 billion neurons in your head has about 1,000 connections with other neurons, creating huge network of about 100 trillion synapses. Like a computer network built from one hundred trillion transistors, each responding a "bit" of information depending on whether it is on or off". Hanson. R. ( 2007) Your Static Wonderful Brain. So in this way we can define the articulation of human's actions through brain. Human brain can process various types of functions and come up with different kinds of decisions. Those decisions could be categorized as good or evil which have some connections with the concept of superpowers.

Keywords: Superpowers, Situations and Relationships, Real life, Negativity, Positivity
*Correspondence to Author:

Farhana Haque

Brac University, Mohakhali, Dhaka,Bangladesh

How to cite this article:

Farhana Haque. Dream of Having Superpowers: Comparison between Good and Bad Superpowers in Real Life as a Person According to Science. American Journal of History and Culture, 2018,1:1.

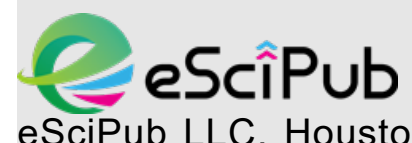

eSciPub LLC, Houston, TX USA. Website: http://escipub.com/ 


\section{Introduction}

In this paper the ingrained meaning about having superpowers basically indicate towards human's abilities in a good and bad situations. As we know different persons carry different types of mind, activities and as a whole different types of energies. They have their own feeling to empower their situations according to their level of confidence and abilities. For example, if anyone dream that he/she can fly, that means he/she dream abut having superpowers. Therefore it implies that you always look at the positives of even a bad situation. You have limitless energies. You are also feeling empowered and confident about your abilities. Consider the significance of your superpower and how it applies to a situation or relationship in your waking life. If you dream that you can fly, then it means that you are on top of the situation. If you dream that you can make things move by waving your hand, then the dream is telling you that you need to put your ideas into action. It could also indicate that things in life have come a little easy for you. If you dream that you can read other people's minds, then it signifies that you have clarity in some situation or problem. You are experiencing peace of mind. If you dream that you can change the weather, then it refers to your ability to turn certain emotions on and off.

\section{According to science what is called superpowers in real life?}

Lets discuss about myself to define "what is called superpowers"? Being a single mother, i do work outside and also do look after my daughter and son. Often i face multiple tasks in order to assist my two kids. Most of the time i see my one child is facing sickness therefore $i$ am in charge of taking care. On the other hands, $i$ do acknowledge my another child's important project in school and there also she needs my help. Not only my children, at home and at my workplace i am bound to fulfil some significant duties such as preparing dinner for all the members of my family then online assignment due mostly at midnight. To make a proper balance between my own life, my children's life and home work, so with great emphasis i feel to name it as superpower. Its the utter strength of me which actually help me to accomplish my each and every tasks as like a superwoman. To manage the pile of duties, sometimes i feel exhausted but then i convince to my existence by saying that, i have to do all those tasks accurately. No matter how much trouble i have to face, eventually i strongly believe, i will surely get success. This is what $i$ call superpower within myself.

In another way, now i am about to vignette the negative sides. The criminals who are known to be the prohibited people and everyone disown them from society. These kinds of people have chosen their life in negative ways which we call as evil ways. They didn't born like a criminal but somehow the situation or lack or knowledge made them criminals. Over time, their simple negative activities become more destructive and dangerous. Like the positive minded people they have the same powerful brain activities. The same power to know or choose which is good or which is bad. But they have chosen their life in a evil way out of frustration and lack of hope.

However, then what about the middle-of-theroad persons, which we can define as midway between extremes. Specially being neither liberal nor conservative. Mostly the general people who doesn't posses those extreme issues such as extreme good or extreme bad. Therefore i think, they need sheer motivation to utilize their internal superpowers in order to uplift them to the higher stage. Apart from those extreme good or evil people, this middle of-theroad persons did set themselves with average outcome in life and become satisfied about what they are or from where they do belong. From every angle they are content. Stacia Pierce described her opinions regarding negativity and positivity of human mind via Huffington post. She indicates, "In life there may be times when negativity seems to surround you, suffocating your dreams and destroying your hope. When that happens, many go through their day being 
saturated with negativity and accepting it as a way of life. While you can't always control what happens to you, you can control how you respond to it, seek to create a positive environment for yourself and you'll begin to become more motivated to achieve your dreams and goals". Pierce. S. ( March 2014) 10 Ways To Stay motivated When Negativity Seems To Be All Around

\section{conclusion}

Since we know that, structure of human beings are magnificently created. Our creator did create us with the balance of logic and emotion which truly lead us to various directions. We are already the amazing creatures on earth who can process and control every kinds of works to

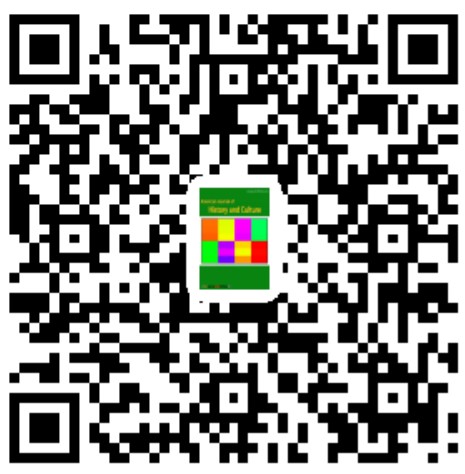
acquire different goals. So it is obvious that we don't need to desire for superpowers. Literally its up to us whether or not to use our instinct superpowers for our good or evil.

\section{References}

1. Hanson. R. ( 2007). Your Static Wonderful Brain. www.wiseBrain.org

2. Pierce. S. ( March 2014). 10 Ways To Stay motivated When Negativity Seems To Be All Around. US: Huffpost 